\title{
Selective lymph node dissection for clinical T1 stage non-small cell lung cancer
}

\author{
Jin-Long Zhao ${ }^{1,2}$, Hong-Wei Guo ${ }^{3}$, Peng Yang ${ }^{2}$, Da-Zhi Jiang ${ }^{2}$, Hui Tian ${ }^{1}$ \\ ${ }^{1}$ Department of Thoracic Surgery, Qilu Hospital of Shandong University, Jinan 250012, China; ${ }^{2}$ Department of Thoracic Surgery, ${ }^{3}$ Department of \\ Pathology, Linyi People's Hospital, Linyi 276000, China \\ Contributions: (I) Conception and design: H Tian; (II) Administrative support: JL Zhao; (III) Provision of study materials or patients: HW Guo; (IV) \\ Collection and assembly of data: P Yang; (V) Data analysis and interpretation: DZ Jiang; (VI) Manuscript writing: All authors; (VII) Final approval of \\ manuscript: All authors. \\ Correspondence to: Hui Tian. Department of Thoracic Surgery, Qilu Hospital of Shandong University, No. 107, Wenhuaxi Road, Lixia District, Jinan \\ 250012, China. Email: tianhuiqlyy@163.com.
}

Background: More and more pulmonary nodules are detected by CT scan, and postoperative pathology reveals many lymph nodes without metastasis. The purpose of this study was to investigate the characteristics of T1 stage lymph node metastasis in non-small cell lung cancer (NSCLC) and to explore the indications for selective lymph node dissection (SLND).

Methods: A total of 841 patients with stage T1 of NSCLC were performed lobectomy and systemic lymphadenectomy. We analyzed the types of lymph node metastases and the relationship between lymph node metastasis and pulmonary pleural invasion, thrombosis of vascular carcinoma and tumor size in all patients.

Results: Among them, 257 cases of tumor in the right upper lobe (RUL) and 186 cases in the left upper lobe (LUL), and no metastasis was found in the inferior mediastinal lymph nodes. Tumor metastases occurred in subcarinal lymph nodes, with hilar and/or mediastinal lymph node metastasis. Among the 171 cases with right lower lobe (RLL) tumors and the 151 cases with left lower lobe (LLL) tumors, patients with superior lymph node metastasis were all associated with hilar and/or subcarinal lymph node metastasis. Among the 76 cases with right middle lobe (RML) tumors, no metastasis with inferior mediastinal lymph node was observed. Lymph node metastasis is much easier in patients with pulmonary pleural invasion or thrombosis of vascular cancer. The larger the tumor diameter, the greater the possibility of lymph node metastasis.

Conclusions: SLND is a feasible treatment for clinical T1 stage NSCLC under the guidance of intraoperative frozen results of lobe-specific lymph nodes.

Keywords: Selective lymph node dissection (SLND); surgery; systematic lymph node dissection; non-small cell lung cancer (NSCLC)

Submitted Jun 28, 2019. Accepted for publication Oct 10, 2019.

doi: $10.21037 /$ tcr.2019.10.46

View this article at: http://dx.doi.org/10.21037/tcr.2019.10.46

\section{Introduction}

Recent advances lung cancer screening and the extensive use of imaging facilities have led to an earlier diagnosis of non-small cell lung cancer (NSCLC) (1). Currently, the treatment methods for lung cancer mainly include surgical treatment, radiotherapy, chemotherapy, molecular targeted therapy and multidisciplinary treatment (2). For patients with T1 stage of NSCLC but without distant metastasis, surgical treatment remains the accepted choice and the only possible cure (3). In clinical practice we carried out selective 
lymph node dissection (SLND) for patients with early-stage NSCLC, although many lymph nodes were dissected, the operative time, postoperative drainage tube indwelling time and postoperative chylothorax incidence were all increased, but the lymph nodes dissected were rarely metastatic (4). In recent years, with the increase of elderly patients with relatively poor body condition and the popularity of "minimally invasive" concept, in order to reduce surgical trauma, many scholars have explored sublobectomy methods, such as anatomical pulmonary segmentectomy or wedge resection to treat stage T1 lung cancer $(5,6)$.

Our study retrospectively analyzed the clinical data of patients with T1 stage of NSCLC to explore the characteristics and patterns of lymph node metastasis (LNM), and designed a more effective method for lymphadenectomy.

\section{Methods}

\section{Patients and methods}

This study enrolled 841 cases of clinical T1-stage NSCLC who underwent lobectomy with systematic lymphadenectomy through thoracotomy or video-assisted thoracoscopic surgery (VATS) from January 2015 to August 2018. All patients were preoperatively diagnosed with a solitary pulmonary nodule less than $3 \mathrm{~cm}$ in diameter by computer tomography scan.

Mediastinal lymph nodes were classified and defined according to the 8 versions of lung cancer TNM staging (7). Mediastinal lymph nodes are grouped by the International Association for the Study of Lung Cancer (8). In the operation, if there is no preoperative pathological diagnosis, the intraoperative pathological diagnosis must be carried out. The ipsilateral thoracic lymph nodes, including N1 and $\mathrm{N} 2$ lymph nodes, were removed. N1 lymph nodes comprise hilar nodes (\#10, \#11, and \#12), N2 lymph nodes include superior mediastinal lymph nodes (SMLN) (\#2R, \#3 and \#4R), subcarinal lymph nodes (SCLN) (\#7) and inferior mediastinal lymph nodes (IMLN) (\#8, \#9). Skip metastasis mainly refers to N2 lymph node metastasis without N1 lymph node metastasis. In all patients, in addition to the hilar lymph nodes, at least three mediastinal lymph nodes including subcarinal lymph nodes (\#7) were removed.

We recorded the gender and age, lymph node metastasis, the location and size of the tumor, the presence of visceral pleural invasion and vascular carcinoma thrombi in all patients, and made statistical analysis of the recorded data.

\section{Statistical analysis}

SPSS software.20.0 was used for statistical analysis (SPSS, Chicago, Illinois, USA), compare frequencies as needed using the chi-square test or the fisher exact test. Bilateral $\mathrm{P}$ value 0.05 is significant.

\section{Results}

Among the 841 cases of clinical T1 stage NSCLC included in this study, 738 cases $(87.8 \%)$ of adenocarcinoma were diagnosed by histopathology, 84 cases $(10.0 \%)$ of squamous cell carcinoma, and the other 19 cases $(2.2 \%)$. The pathological degree of LNM was N0 in 749 cases (89.1\%), N1 in 66 cases (7.8\%), and N2 in 60 cases (7.1\%). Right upper lobe (RUL) lung tumor is 257 cases (30.6\%), right middle lobe (RML) lung tumor is 76 cases $(9.0 \%)$, right lower lobe (RLL) lung tumor is 171 cases $(20.3 \%)$, left upper lobe (LUL) lung tumor is 186 cases $(22.1 \%)$ and left lower lobe (LLL) lung tumor is 151 cases $(18.0 \%)$. The location of primary lesion, LNM and pathological diagnosis were shown in Table 1.

\section{Lymph node metastasis depends on the location of the primary tumor}

\section{LNM in 504 cases with right lung tumors Right upper lobe (Table 2)}

A total of 257 cases of the primary tumor were in the RUL, and 25 cases $(9.7 \%)$ had lymph node metastasis. Eight cases had skip lymph node metastasis, including 7 cases with single metastasis to the superior mediastinum (\#2-4) and one case with both superior mediastinum and subcarinal (\#7) metastasis.

There were no cases of inferior mediastinal metastasis (\#8-9).

\section{Right middle lobe (Table 3)}

A total of 76 cases had primary tumors in the RML, and lymph node metastasis in 5 cases $(6.6 \%)$. Two cases presented skip lymph node metastasis, including one case with metastasis to superior mediastinum and one case with both superior mediastinum and subcarinal metastasis. No cases showed IMLN metastasis.

\section{Right lower lobe (Table 4)}

There were 171 cases of primary right inferior lobe tumor and 20 cases of LNM (11.7\%). There were 6 cases had skip metastasis, including 1 case with metastasis to the inferior mediastinum, 4 cases with only metastasis to the subcarina, 
Table 1 Characteristics of patients with clinical T1 stage NSCLC

\begin{tabular}{|c|c|c|}
\hline Characteristics & Number & $\%$ \\
\hline \multicolumn{3}{|l|}{ Sex } \\
\hline Male & 371 & 44.1 \\
\hline Female & 470 & 55.9 \\
\hline \multicolumn{3}{|l|}{ Histologic type } \\
\hline Adenocarcinoma & 738 & 87.8 \\
\hline Squamous cell carcinoma & 84 & 10.0 \\
\hline Others & 19 & 2.2 \\
\hline \multicolumn{3}{|l|}{ Tumor size $(\mathrm{cm})$} \\
\hline$\leq 1.0$ & 177 & 21.0 \\
\hline$>1.0$ to $\leq 2.0$ & 404 & 48.0 \\
\hline$>2.0$ to $\leq 3.0$ & 260 & 30.9 \\
\hline \multicolumn{3}{|l|}{ Postoperative $\mathrm{N}$ status } \\
\hline NO & 749 & 89.1 \\
\hline N1 & 66 & 7.8 \\
\hline N2 & 60 & 7.1 \\
\hline \multicolumn{3}{|l|}{ Tumor location } \\
\hline Right upper lobe & 257 & 30.6 \\
\hline Right middle lobe & 76 & 9.0 \\
\hline Right lower lobe & 171 & 20.3 \\
\hline Left upper lobe & 186 & 22.1 \\
\hline Left lower lobe & 151 & 18.0 \\
\hline \multicolumn{3}{|l|}{ Post-operative staging } \\
\hline I & 749 & 89.1 \\
\hline la & 749 & 89.1 \\
\hline $\mathrm{lb}$ & 0 & 0 \\
\hline II & 66 & 7.8 \\
\hline Ila & 0 & 0 \\
\hline $\mathrm{Ilb}$ & 66 & 7.8 \\
\hline Illa & 60 & 7.1 \\
\hline \multicolumn{3}{|l|}{ Pleural involvement } \\
\hline Pleura involved & 273 & 32.5 \\
\hline Pleura not involved & 568 & 67.5 \\
\hline
\end{tabular}

and 1 case of simultaneous metastasis with both superior mediastinum and subcarina. All patients with SMLN metastases also had hilar and/or SCLN metastases.

\section{LNM in 337 patients with left lung tumor}

\section{Left upper lobe (Table 5)}

There were 186 cases of primary left upper lobe tumor and 21 cases $(11.3 \%)$ had lymph node metastasis. Six cases had skip metastasis, all with SMLN metastases, but none with IMLN or SCLN metastasis.

\section{Left lower lobe (Table 6 )}

There were 151 cases of primary left lower lobe tumor and 21 cases $(13.9 \%)$ had LNM. Four cases had skip metastasis, including two cases with simple SCLN metastasis and two cases with simple IMLN metastasis. All patients with SMLN metastasis had hilar lymph node metastasis, with or without SCLN metastasis.

\section{Skip N2 cases}

In our cases, 26 patients (3.1\%) had skip N2; RUL 8/257, 3.1\%; RML 2/76, 2.6\%; RLL 6/171, 3.5\%; LUL 6/186, $3.2 \%$; LLL 4/151, 2.6\% (Table 7); Our study found that the possibility of lymph node skip metastasis of lung cancer was not correlated with the primary location of tumor $(\mathrm{P}=0.998)$. However, lymph nodes are more likely to jump to the upper mediastinum for tumors in the upper lobe, while lymph nodes are more likely to jump to subcarinal and the inferior mediastinum for tumors in the lower lobe.

\section{Relationship between LNM and lung pleural invasion}

Among the 841 patients, 273 cases had tumor invasion to the visceral pleura and 56 cases $(20.51 \%)$ had lymph node metastasis. There were 568 cases without tumor invasion in lung pleura, among which 36 cases $(6.33 \%)$ had LNM. The metastasis rate of patients with lung pleural tumor invasion was higher than that without invasion $\left(\chi^{2}=38.03, \mathrm{P}<0.01\right)$, indicating that patients with visceral pleural invasion are more likely to have LNM (Table 8).

\section{Relationship between LNM and vascular carcinoma thrombi}

Of the 841 patients, 15 had vascular thrombosis, including 13 with adenocarcinoma and 2 with squamous cell carcinoma. 10 of the cases $(66.67 \%)$ showed lymph node metastasis (Table 9); all cases were accompanied with hilar lymph node metastasis and 8 cases with SMLN or SCLN metastasis, but no cases had IMLN metastasis or skip metastasis. The metastasis rate in patients with vascular carcinoma was significantly higher than that of patients without vascular carcinoma thrombi $\left(\chi^{2}=43.03, \mathrm{P}<0.01\right)$, 
Table 2 Lymph node metastasis in 257 cases with right upper lung tumors

\begin{tabular}{|c|c|c|c|c|c|c|c|c|}
\hline LN station & \multicolumn{8}{|c|}{ Right upper lobe tumors $(\mathrm{n}=257)$} \\
\hline \# 2-4 & - & - & + & - & + & + & + & 18 \\
\hline$\# 7$ & - & - & - & + & + & + & - & 4 \\
\hline \# 8-9 & - & - & - & - & - & - & - & 0 \\
\hline Total & 232 & 5 & 9 & 2 & 1 & 1 & 7 & \\
\hline
\end{tabular}

LN, lymph node; +, lymph node with metastasis; -, lymph node without metastasis.

Table 3 Lymph node metastasis in 76 cases with right middle lung tumors

\begin{tabular}{|c|c|c|c|c|c|c|}
\hline LN station & \multicolumn{6}{|c|}{ Right middle lobe tumors $(n=76)$} \\
\hline \# 2-4 & - & - & - & + & + & 2 \\
\hline$\# 7$ & - & - & + & - & + & 3 \\
\hline \# 8-9 & - & - & - & - & - & 0 \\
\hline Total & 71 & 1 & 2 & 1 & 1 & \\
\hline
\end{tabular}

LN, lymph node; +, lymph node with metastasis; -, lymph node without metastasis.

Table 4 Lymph node metastasis in 171 cases with right lower lung tumors

\begin{tabular}{|c|c|c|c|c|c|c|c|c|c|}
\hline LN station & \multicolumn{9}{|c|}{ Right lower lobe tumors $(n=171)$} \\
\hline \#2-4 & - & - & + & - & + & - & - & + & 6 \\
\hline \#7 & - & - & - & + & + & - & + & + & 11 \\
\hline \#8-9 & - & - & - & - & - & + & - & - & 1 \\
\hline Total & 151 & 6 & 2 & 3 & 3 & 1 & 4 & 1 & \\
\hline
\end{tabular}

LN, lymph node; +, lymph node with metastasis; -, lymph node without metastasis.

suggesting that lymph node metastasis is more easier to occur in patients with vascular cancer and thrombosis, and lymph node metastasis is likely to follow the pattern of lymphatic drainage (from N1 lymph node to N2 lymph node), and jumping metastasis is rare.

\section{Relationship between LNM and tumor size}

Among the 841 patients, there were 177 patients with tumors $0-1 \mathrm{~cm}$ in diameter (Group A), and 1 case $(0.56 \%)$ had lymph node metastasis; 404 patients had tumors of 1-2 cm (Group B), and 22 cases (5.45\%) had LNM; 260 patients had tumors of $2-3 \mathrm{~cm}$ (Group C), and 69 cases (26.54\%) had lymph node metastasis. There was statistically significant difference in the transfer rate between the two groups: Group A vs. Group B $\left(\chi^{2}=7.71, \mathrm{P}<0.01\right)$; Group B vs. Group $\mathrm{C}\left(\chi^{2}=59.51, \mathrm{P}<0.01\right)$; and Group A vs. Group $\mathrm{C}\left(\chi^{2}=52.81, \mathrm{P}<0.01\right)($ Table 10$)$. These results suggest that the larger the tumor diameter, the greater the likelihood of lymph node metastasis. 
Table 5 Lymph node metastasis in 186 cases with left upper lung tumors

\begin{tabular}{|c|c|c|c|c|c|c|c|c|}
\hline LN station & \multicolumn{8}{|c|}{ Left upper lobe tumors $(n=186)$} \\
\hline$\# 4 \mathrm{~L}$ & - & - & + & - & + & + & - & 5 \\
\hline$\# 5-6$ & - & - & - & + & + & - & + & 8 \\
\hline$\# 7$ & - & - & - & - & - & - & - & 0 \\
\hline \#10-12 & - & + & + & + & - & - & - & 14 \\
\hline Total & 166 & 10 & 2 & 3 & 2 & 1 & 3 & \\
\hline
\end{tabular}

LN, lymph node; +, lymph node with metastasis; -, lymph node without metastasis.

Table 6 Lymph node metastasis in 151 cases with left lower lung tumors

\begin{tabular}{|c|c|c|c|c|c|c|c|c|c|}
\hline \multirow{2}{*}{ LN station } & \multicolumn{9}{|c|}{ Left lower lobe tumors $(n=151)$} \\
\hline & Mode 1 & Mode 2 & Mode 3 & Mode 4 & Mode 5 & Mode 6 & Mode 7 & Mode 8 & Total \\
\hline$\# 4 \mathrm{~L}$ & - & - & - & - & + & + & - & - & 2 \\
\hline \#5-6 & - & - & + & + & - & - & - & - & 4 \\
\hline$\# 7$ & - & - & + & - & - & + & - & + & 4 \\
\hline \#10-12 & - & + & + & + & + & + & - & - & 17 \\
\hline Total & 130 & 11 & 1 & 3 & 1 & 1 & 2 & 2 & \\
\hline
\end{tabular}

LN, lymph node; +, lymph node with metastasis; -, lymph node without metastasis.

Table 7 Skip N2 metastasis

\begin{tabular}{|c|c|c|c|c|c|}
\hline LN station & \multicolumn{5}{|c|}{ Tumor location } \\
\hline \#2-4 & 8 & 3 & 1 & 1 & \\
\hline \#5-6 & & 5 & & & \\
\hline$\# 7$ & 1 & & 1 & 5 & 2 \\
\hline$\chi^{2}$ & & & 0.263 & & \\
\hline$P$ & & & 0.998 & & \\
\hline
\end{tabular}

LN, lymph node; RUL, right upper lung; LUL, left upper lung; RML, right middle lung; RLL, right lower lung; LLL, left lower lung.

Table 8 Relationship between lymph node metastases and visceral pleural invasion

\begin{tabular}{lccc}
\hline Visceral pleural invasion & $\mathrm{N}$ & Lymph node metastasis, n (\%) & Without lymph node metastasis, n (\%) \\
\hline Involved & 273 & $56(20.51)$ & $217(79.48)$ \\
Uninvolved & 568 & $36(6.33)$ & $532(93.66)$ \\
$\chi^{2}$ & & 38.03 \\
$P$ & & $<0.01$ \\
\hline
\end{tabular}


Table 9 Relationship between lymph node metastasis and vascular carcinoma thrombi

\begin{tabular}{lccc}
\hline Vascular carcinoma thrombi & $\mathrm{N}$ & Lymph node metastasis, $\mathrm{n}(\%)$ & Without lymph node metastasis, $\mathrm{n}(\%)$ \\
\hline With & 15 & $10(66.67)$ & $5(33.33)$ \\
Without & 826 & $82(9.93)$ & $744(90.07)$ \\
$\chi^{2}$ & & 43.03 & $<0.01$ \\
$\mathrm{P}$ & & & \\
\hline
\end{tabular}

Table 10 Relationship between lymph node metastasis and tumor size

\begin{tabular}{lccc}
\hline Tumor size, cm & $\mathrm{N}$ & Lymph node metastasis, $\mathrm{n}(\%)$ & Without lymph node metastasis, $\mathrm{n}(\%)$ \\
\hline $0-1$ & 177 & $1(0.56)$ & $176(99.44)$ \\
$1-2$ & 404 & $22(5.45)$ & $382(94.55)$ \\
$2-3$ & 260 & $69(26.54)$ & $191(73.46)$ \\
$\chi^{2}$ & & & 97.006 \\
$\mathrm{P}$ & & $<0.01$ \\
\hline
\end{tabular}

\section{Discussion}

The principle of surgical treatment for localized NSCLC is to retain the function of normal tissues and organs to the maximum extent on the basis of complete resection of the lesion lobectomy or systemic mediastinal pneumonectomy and hilar lymph node resection, known as radical SLND. Mediastinal LNM is one of the most important adverse prognostic factors, accounting for about $20-40 \%$ of NSCLC patients (9). However, the incidence of pulmonary small nodules has been gradually increasing. It is also important to remove metastatic lymph nodes while retaining intact ones. We need to rethink routine lymph node dissection. Therefore, we discussed the possibility of reducing the degree of SLND.

A lot of previous studies have shown that the location of the primary tumor affects the extent of nodule spread (10-12). Okada and his colleagues reported that none of the patients with skipped N2 metastasis (no N1 nodes involved) with an upper-lobe lesion had a positive subtrochanteric lymph node (10). Of the 13 lower lobar lesions, only 1 (7.7\%) had nodules that spread up the mediastinum. In the authors' opinion, if the hilar and superior mediastinal lymph nodes of the upper lobe tumor are non-neoplastic, then the lower mediastinal lymph nodes do not need to be removed. If the hilar and subtrochanteric lymph nodes are not cancerous, the superior mediastinal lymph nodes need to be removed. This study suggested that selective lymphadenectomy based on a leaf-specific pattern of lymph node metastasis is effective.

Lymph node dissection was performed according to the model theory of large leaf specific LNM, when the tumor is located in the RUL, the SMLN (\#2R, \#3, \#4R) and hilar nodes $(\# 10, \# 11, \# 12)$ are removed. But when the tumor is located in the LUL, the SMLN (\#4L, \#5, \#6) in addition to hilar nodes $(\# 10, \# 11, \# 12)$ are resected. In these cases, no inferior mediastinal dissection was performed in the intraoperative diagnosis of hilar and superior mediastinal lymph node metastasis. In cases where lymph node metastasis is suspected, a macroscopic analysis of intraoperative frozen sections is performed, and if positive, these patients will undergo a systematic lymph node dissection. In addition, when the tumor is located in the inferior lobe, subcarinal (\#7) and submediastinal (\#8, \#9) lymph nodes are removed, and superior mediastinal lymph nodes are removed when SCLN and IMLN are negative intraoperatively. By such definition, among our 841 patients, all metastatic lymph nodes should be completely removed. We found that there must be hilar or mediastinal LNM in the upper lobe of the lung with subcarinal lymph node metastasis. So, we can use lobe-specific lymph nodes as sentinel nodes to determine whether or not to do systematic lymph node dissection.

If we only detected hilar lymph nodes by intraoperative frozen sections and determines the mode of lymph node dissection, we found that two cases $(0.24 \%)$ would have 
cancer cell residues due to SLND. One case was an adenocarcinoma of the right lower lobe. The tumor size was $2.2 \mathrm{~cm} \times 2.0 \mathrm{~cm}$, acinar dominant type, with visceral pleural invasion, CT scan revealed pure solid components; there were SMLN and SCLN metastases but no hilar lymph node metastasis. In this case, SLND may result in superior mediastinal cancer cell residue. Here is another case of medially differentiated squamous cell carcinoma of the right upper lobe, the tumor size was $2.0 \mathrm{~cm} \times 2.0 \mathrm{~cm}$, CT scan revealed pure solid components; there were SMLN and SCLN metastases but no hilar lymph node metastasis. In this case, SLND would lead to subcarinal cancer cell residue. So, using hilar lymph nodes as sentinel nodes alone can lead to metastatic lymph nodes residual.

Few studies have been reported on the pattern of LNM in the middle lobe of right lung. Asamura et al. (12) and Watanabe et al. (13) showed that the tumor in the RML had no specific pattern of lymphatic spread. In our study, among the 76 patients with tumors in the RML, 5 cases $(6.58 \%)$ had lymph node metastases, including two cases with SMLN metastases and three cases with SCLN metastases, but no inferior lymph node metastasis, indicating that tumors in the right middle lobe are prone to SMLN and SCLN metastasis.

Based on the above analysis, we suggest that for clinical T1 stage non-right middle lobe NSCLC, SLND is a feasible treatment according to the intraoperative frozen results of lobe-specific lymph nodes; For clinical T1 stage right middle lobe NSCLC patients, we suggest that hilar lymph nodes, SMLN and SCLN should be dissected, and the dissection of the IMLN can be omitted; SLND is recommended for lung tumors larger than $2 \mathrm{~cm}$ in diameter with simple solid components or visceral pleural infiltration.

Although lymph node dissection is helpful in determining the histologic staging of lymph node spread, it has no effect on overall or disease-free survival of lymph nodes $(14,15)$. Lymph node dissection without cancer cells is considered ineffective, may prolong operative time and increase perioperative complications (14-17). Ishiguro et al. (14) found that the operation time of SLND patients was shorter than the patients with systematic lymph node dissection, with less blood loss during surgery and shorter hospital stays, indicating that SLND was less invasive. In Japan, SLND is commonly used for people with poor physical conditions and early disease (14).

Hishida et al. showed that lobe-specific lymph node dissection (LSD) had no negative effect on prognosis in patients with c-stage I-II of NSCLC (18). The 5-year overall survival rate of the LSD group was $81.5 \%$ and $75.9 \%$ in the systematic lymph node dissection group. LSD was associated with good survival (hazard ratio $=0.68,95 \%$ CI: 0.60-0.77). Okada pointed out that the treatment effect of selective mediastinal dissection on NSCLC was the same as that of complete dissection, and there was no difference in disease-free survival and overall survival between the two groups (19). Several randomized studies have shown that completion of systematic lymph node dissection has no therapeutic effect compared to systematic sampling (20-23). In addition, circulating tumor cells are common in early cancers (24-26). Thus, these data informed that lymph nodal involvement is a marker of metastatic predisposition and that removal of lymph nodes does not prevent the development of metastasis.

It was reported that lymph node metastasis was significantly associated with large tumor size, solid components, lymphovascular invasion and visceral pleural invasion in clinical stage IA NSCLC $(27,28)$. Our study also presented the similar result that lymph node metastasis was much easier in patients with pulmonary pleural invasion or thrombosis of vascular cancer. The larger the tumor diameter, the greater the possibility of lymph node metastasis.

\section{Conclusions}

To sum up, minimally invasive surgery is an excellent treatment for early NSCLC, and lob-specific lymph node resection is feasible in practice. We recommend that for clinical T1 stage non-right middle lobe NSCLC, SLND is a feasible treatment according to the intraoperative frozen results of lobe-specific lymph nodes; For clinical T1 stage right middle lobe NSCLC patients, we suggest that hilar lymph nodes, SMLN and SCLN should be dissected, and the dissection of the IMLN can be omitted; For lung tumors larger than $2 \mathrm{~cm}$ in diameter with pure solid components or visceral pleura invasion, systematic lymph node dissection is recommended.

\section{Acknowledgments}

Funding: This work was supported by the national natural science foundation of China (81672292); Taishan Scholar Program of Shandong Province (No. ts201712087); and the Department of Pathology of Linyi People's Hospital, Linyi City, Shandong Province, China. 


\section{Footnote}

Conflicts of Interest: All authors have completed the ICMJE uniform disclosure form (available at http://dx.doi. org/10.21037/tcr.2019.10.46). The authors have no conflicts of interest to declare.

Ethical Statement: The authors are accountable for all aspects of the work in ensuring that questions related to the accuracy or integrity of any part of the work are appropriately investigated and resolved. The study was conducted in accordance with the Declaration of Helsinki (as revised in 2013). The study protocol was approved by Medical Ethics Committee of Linyi People's Hospital (No. 30039), and we assure that all participants were given informed consent before taking part.

Open Access Statement: This is an Open Access article distributed in accordance with the Creative Commons Attribution-NonCommercial-NoDerivs 4.0 International License (CC BY-NC-ND 4.0), which permits the noncommercial replication and distribution of the article with the strict proviso that no changes or edits are made and the original work is properly cited (including links to both the formal publication through the relevant DOI and the license). See: https://creativecommons.org/licenses/by-nc-nd/4.0/.

\section{References}

1. Torre LA, Bray F, Siegel RL, et al. Global cancer statistics, 2012. CA Cancer J Clin 2015;65:87-108.

2. Kodama K, Higashiyama M, Okami J, et al. Oncologic Outcomes of Segmentectomy Versus Lobectomy for Clinical T1a N0 M0 Non-Small Cell Lung Cancer. Ann Thorac Surg 2016;101:504-11.

3. Hwang Y, Kang CH, Kim HS, et al. Comparison of thoracoscopic segmentectomy and thoracoscopic lobectomy on the patients with non-small cell lung cancer: a propensi score matching study. Eur J Cardiothorac Surg 2015;48:273-8.

4. Ou SH, Zell JA. Prognostic significance of the number of lymph nodes removed at lobectomy in stage IA non-small cell lung cancer. J Thorac Oncol 2008;3:880-6.

5. Schuchert MJ, Abbas G, Awais O, et al. Anatomic segmentectomy for the solitary pulmonary nodule and early-stage lung cancer. Ann Thorac Surg 2012;93:1780-5.

6. Nakamura H, Taniguchi Y, Miwa K, et al. Comparison of the surgical outcomes of thoracoscopic lobectomy, segmentectomy, and wedge resection for clinical stage I non-small cell lung cancer. Thorac Cardiovasc Surg 2011;59:137-41.

7. Brierley J, Gospodarowicz MK, Wittekind CH. TNM classification of malignant tumours. 8th edition. Chichester, West Sussex, UK; Systematic lymph node dissection was recommended for the lung tumors larger than $2 \mathrm{~cm}$ in diameter accompanied with visceral pleura invasion. Hoboken, NJ: John Wiley \& Sons, Inc., 2017; 106-12.

8. Rusch VW, Asamura H, Watanabe H, et al. The IASLC lung cancer staging project: a proposal for a new international lymph node map in the forthcoming seventh edition of the TNM classification for lung cancer. J Thorac Oncol 2009;4:568-77.

9. Mountain CF. A new international staging system for lung cancer. Chest 1986;89:225S-33S.

10. Okada M, Tsubota N, Yoshimura M, et al. Proposal for reasonable mediastinal lymphadenectomy in bronchogenic carcinomas: role of subcarinal nodes in selective dissection. J Thorac Cardiovasc Surg 1998;116:949-53.

11. Watanabe $Y$, Shimizu J, Tsubota M, et al. Mediastinal spread of metastatic lymph nodes in bronchogenic carcinoma. Mediastinal nodal metastases in lung cancer. Chest 1990;97:1059-65.

12. Asamura H, Nakayama H, Kondo H, et al. Lobe-specific extent of systematic lymph node dissection for non-small cell lung carcinomas according to a retrospective study of metastasis and prognosis. J Thorac Cardiovasc Surg 1999;117:1102-11.

13. Watanabe S, Asamura H, Suzuki K, et al. The new strategy of selective nodal dissection for lung cancer based on segment-specific patterns of nodal spread. Interact Cardiovasc Thorac Surg 2005;4:106-9.

14. Ishiguro F, Matsuo K, Fukui T, et al. Effect of selective lymph node dissection based on patterns of lobe-specific lymph node metastases on patient outcome in patients with resectable non-small cell lung cancer: A large-scale retrospective cohort study applying a propensity score. J Thorac Cardiovasc Surg 2010;139:1001-6.

15. Adachi H, Sakamaki K, Nishii T, et al. Lobe-Specific Lymph Node Dissection as a Standard Procedure in Surgery for Non-Small Cell Lung Cancer: A Propensity Score Matching Study. J Thorac Oncol 2017;12:85-93.

16. Kuroda H, Sakao Y, Mun M et al. Lymph Node Metastases and Prognosis in Left Upper Division Non-Small Cell Lung Cancers: The Impact of Interlobar Lymph Node 
Metastasis. PLoS One 2015;10:e0134674.

17. Lardinois D, Suter H, Hakki H, et al. Morbidity, survival, and site of recurrence after mediastinal lymph-node dissection versus systematic sampling after complete resection for non-small cell lung cancer. Ann Thorac Surg 2005;80:268-74.

18. Hishida T, Miyaoka E, Yokoi K, et al. Lobe-Specific Nodal Dissection for Clinical Stage I and II NSCLC: Japanese Multi-Institutional Retrospective Study Using a Propensity Score Analysis. J Thorac Oncol 2016;11:1529-37.

19. Okada M, Sakamoto T, Yuki T, et al. Selective mediastinal lymphadenectomy for clinico-surgical stage I non-small cell lung cancer. Ann Thorac Surg 2006;81:1028-32.

20. Kim AW, Detterbeck F. Mediastinal lymph node dissection. In: Franco K, Thourani V, eds. Cardiothoracic Surgery Review. Philadelphia, PA: Lippincott Williams and Wilkins; 2012: 1019-22.

21. Allen MS, Darling GE, Pechet TTV, et al. Morbidity and mortality of major pulmonary resections in patients with early-stage lung cancer: initial results of the randomized, prospective ACOSOG Z0030 trial. Ann Thorac Surg 2006;81:1013-19.

22. Sugi K, Nawata K, Fujita N, et al. Systematic lymph node

Cite this article as: Zhao JL, Guo HW, Yang P, Jiang DZ, Tian H. Selective lymph node dissection for clinical T1 stage non-small cell lung cancer. Transl Cancer Res 2019;8(8):2820-2828. doi: $10.21037 /$ tcr.2019.10.46 dissection for clinically diagnosed peripheral non-small cell lung cancer less than $2 \mathrm{~cm}$ in diameter. World J Surg 1998;22:290-4.

23. Wu Y, Huang ZF, Wang SY, et al. A randomized trial of systematic nodal dissection in resectable non-small cell lung cancer. Lung Cancer 2002;36:1-6.

24. Alix-Panabières C, Riethdorf S, Pantel K. Circulating tumor cells and bone marrow micrometastasis. Clin Cancer Res 2008;14:5013-21.

25. Allard WJ, Matera J, Miller MC, et al. Tumor cells circulate in the peripheral blood of all major carcinomas but not in healthy subjects or patients with nonmalignant diseases. Clin Cancer Res 2004;10:6897-904.

26. Castaldo G, Tomaiuolo R, Sanduzzi A, et al. Lung cancer metastatic cells detected in blood by reverse transcriptasepolymerase chain reaction and dot-blot analysis. J Clin Oncol 1997;15:3388-93.

27. Ding N, Mao Y, Gao S, et al. Predictors of lymph node metastasis and possible selective lymph node dissection in clinical stage IA non-small cell lung cancer. J Thorac Dis 2018;10:4061-8.

28. Kudo Y, Saji H, Shimada Y, et al. Impact of visceral pleural invasion on the survival of patients with non-small cell lung cancer. Lung Cancer 2012;78:153-60. 